\title{
DISCONTINUOUS NANOPOROUS STRUCTURES ENABLE LOW-POWER ELECTROPHORETIC IMMUNOASSAYS OF SERUM PROTEIN BIOMARKERS
}

\author{
Chenlu Hou and Amy E. Herr* \\ University of California, Berkeley, CA 94720, USA
}

\begin{abstract}
To realize low-power electrophoretic immunoassays for protein biomarkers, we introduce discontinuous nanoporous structures photopatterned within microfluidic channels as separation matrices. The discontinuous cross-linked gels enhance analyte mobility differences, therefore reducing the required electrophoretic separation length and electrical potential to complete biomarker detection. An optimized mask-based fabrication protocol is introduced to eliminate protein exclusion and "destacking" dispersion near the pore-size discontinuity. Using the optimized sieving matrix, concurrent quantitation of $\mathrm{C}$ reactive protein (CRP) and tumor necrosis factor - alpha (TNF- $\alpha$ ) were developed. Both protein species are important in host inflammatory and infection response. The ultra-short separation distance electrophoretic immunoassay reported here provides flexibility in field-portable diagnostic instrument design by relaxing electrical potential requirements and providing multiplexing capabilities.
\end{abstract}

\section{INTRODUCTION}

Commercially available lateral flow assays - including home pregnancy tests - provide simple "yes/no" readouts. The nonquantitative readouts from such "test strips" cannot provide an accurate representation of complex physiological conditions. Adapting laboratory-grade diagnostic assays for clinical or nearpatient settings would therefore enable rapid clinical decision making, effective disease treatment and containment, and assessment of treatment efficacy.

Many types of assays capable of quantifying protein biomarkers have been developed and employed for clinical diagnostics. To isolate biomarkers of interest, surface-based biosensors require surface immobilization of a capture probe and multiple washing steps. On the other hand, electrophoretic immunoassays rely on effective electrophoretic separation of a probe (antibody) from the larger immune-complex (probe bound to target protein biomarker) in which the magnitude of immunecomplex formation reflects target biomarker concentration. Elimination of surface immobilization and washing steps not only simplifies assay implementation and reduces reagent consumption, but also renders the assay format reusable and appropriate for continuous monitoring. When implemented with microfluidic technology, electrophoretic immunoassays can be automated and integrated with upstream sample preparation.

Yet, a major limitation to point-of-care use of electrophoretic immunoassays centers on the need for high electric fields $(100 \mathrm{~s} \mathrm{~V} / \mathrm{cm})$ applied across centimeters of separation length. Thus, a reduction in the required separation length would, in turn, reduce applied electrical potential (voltage) constraints, while allowing high electric field strength operation.

Our group and others have improved electrophoretic immunoassay performance by fabricating nanoporous sieving matrices within microfluidic separation channels [1]. The sieving matrices enhance mobility differences among analytes, thus reducing the separation distance required for resolving the immune complex. In particular, photopolymerization yields cross-linked polyacrylamide (PA) gels with control over characteristics such as gel pore-size and pore-size distribution. Our previous reports utilized cross-linked polyacrylamide separation gels with moderate gradients in sieving pore-size to complete immunoassays in $1 \mathrm{~mm}$ [2].

We now introduce microchip nanoporous discontinuous gels as an electronic alternative to performance-limited test strips, encompassing both low-power consumption and quantitative biomarker measurement. Our multi-analyte assays build on a new design strategy that obviates cumbersome, expensive high-voltage power supplies currently required for electrophoresis. The approach significantly reduces electrophoretic immunoassay power consumption ( $\mathrm{mW}$ to $\mu \mathrm{W}$ ); consequently, conventional- or solarbattery power is an option for portable operation including quantitative global health, military, and clinical diagnostics.

\section{EXPERIMENTAL \\ Gel Fabrication}

Three separation gel formats were fabricated within microfluidic channel networks as shown in Figure 1A and used to optimize electrophoretic assay performance: 1) uniform pore-size gels, 2) large-to-small pore-size gradient gels, and 3) discontinuous pore-size gels. For all three formats, the microfluidic channels were coated with a self-assembled silane monolayer, which provides covalent linkage of the PA gel to the channel walls. Gel precursor solutions of appropriate acrylamide concentration (indicated as $\% \mathrm{~T})$ were prepared by diluting $30 \%(\mathrm{w} / \mathrm{v})$ acrylamide/bis-acrylamide stock solution with $1 \mathrm{x}$ Tris-glycine electrophoresis buffer to a total volume containing $0.2 \%(\mathrm{w} / \mathrm{v})$ VA086 photoinitiator. Following vigorous sonication and degassing, precursor solutions were introduced into the channels via capillary action. Photopatterning of uniform pore-size gels and decreasing pore-size gradient gels has been previously reported by our group and others $[1,3]$.

To create a large-to-small pore-size discontinuity in the separation channel, a chip filled with high $\% \mathrm{~T}$ precursor solution was aligned to a transparency mask exposing the separation channel to UV illumination from an Olympus IX-50 microscope equipped with a mercury arc lamp and an UV objective. Further details on exposure conditions to optimize the discontinuous gel structure for separation are included in the Results and Discussion section. Following the first exposure, unpolymerized high $\% \mathrm{~T}$ gel precursor solution in the loading channel was flushed out and replaced with low $\% \mathrm{~T}$ precursor solution via pressure driven flow, followed by flood exposure of the entire chip to a UV lamp. As a result, the loading channel was filled with large pore-size gel and a large-to-small pore-size discontinuity was located in proximity to the injection junction in the separation channel.

\section{Gel Pore-size Characterization}

To characterize effective pore-size of discontinuous gels along the separation axis, electrophoretic mobility of a model analyte (anti-C reactive protein antibody) was obtained as a function of migration distance from separation images captured on an Olympus IX-70 microscope equipped with a CCD camera. Mobility measurement of the same model analyte on five uniform pore-size PA gels yields a calibration curve between apparent 


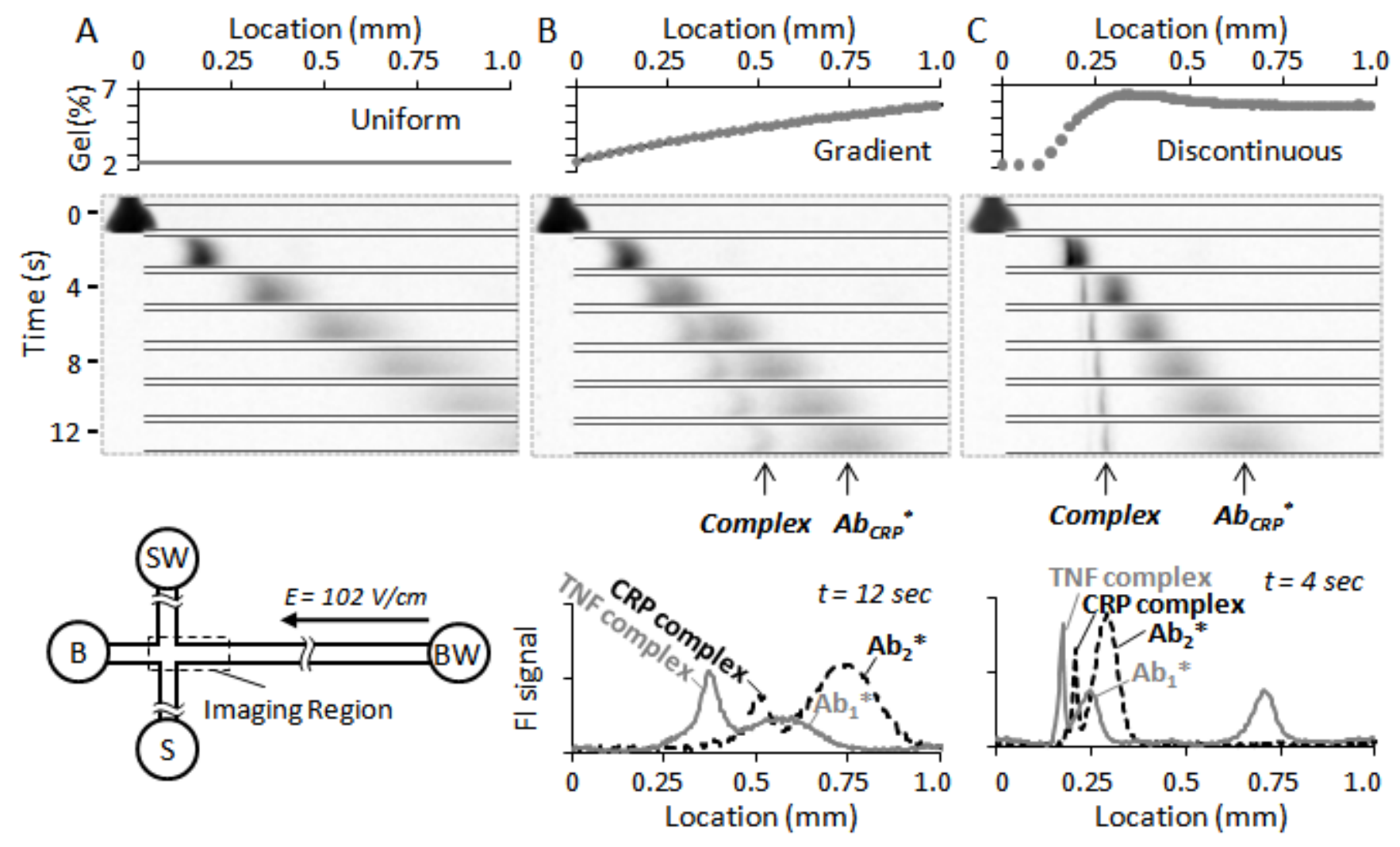

Figure 1. Polymer photopatterning enables unprecedented efficiency $(<5 s,<500$ um) in electrophoretic immunoassays for two protein inflammation markers (CRP, $\mathbf{T N F}-\boldsymbol{\alpha})$. The spatially varying gel pore-size is shown on the top panel, as determined by Ab ${ }_{C R P}{ }^{*}$ mobility. For display clarity, CCD images from the CRP assay with red fluorescence are shown in grayscale for the first $12 \mathrm{~s}$ of the separation (middle panel). Gradient gel fluorescence intensity profiles are shown at $12 \mathrm{~s}$ of separation for the 2-color concurrent assays (B, bottom panel). The concurrent assays achieve similar separation resolution on the discontinuous gel at $4 \mathrm{~s}$ of separation (C, bottom panel). E = $102 \mathrm{~V} / \mathrm{cm}$. Sample contains $16 \mathrm{nM} C R P$ and $22 \mathrm{nM} T N F-\alpha$.

electrophoretic mobility and gel composition (\%T): log (mobility, $\left.\mathrm{cm}^{2} / \mathrm{V} / \mathrm{s}\right)=-0.111(\mathrm{~T})-3.71\left(\mathrm{R}^{2}=0.974\right)$. The calibration curve is used to extract effective gel pore-size along the separation axis for discontinuous gels as well as gradient pore-size gels.

\section{Protein Samples and Assay Setup}

For multi-analyte assays, affinity reagents toward different analytes were labeled with spectrally distinct fluorophores: antiCRP antibody was labeled with Alexa Fluor 568 dyes with red fluorescence and anti-TNF- $\alpha$ antibody was labeled with Alexa Fluor 488 dyes with green fluorescence. Samples containing fluorescently labeled antibodies and target analytes were mixed and incubated in plastic tubes previously coated with a $5 \%(\mathrm{w} / \mathrm{v})$ bovine serum albumin (BSA) solution. The BSA coating minimizes non-specific adsorption of protein samples onto the tube walls. For assay development, the chip was sandwiched between an in-house machined manifold and an aluminum frame for fluid introduction. The SW, B, BW reservoirs were filled with Trisglycine native buffer and a $5 \mu \mathrm{L}$ protein sample was introduced to the $\mathrm{S}$ reservoir. Sample injection and separation was initiated by applying voltage through platinum electrodes inserted in each reservoir.

\section{RESULTS AND DISCUSSION}

Electrophoretic immunoassay is complete when the antibody (probe) peak is resolved from the immune complex peak. The separation is quantified as the separation resolution (SR). SR is defined as the ratio of peak-to-peak separation distance $(\Delta L)$ to peak width $(4 \sigma)$. Assuming uniform electric field and electrophoretic mobility for simplicity, the peak-to-peak distance between the immune complex and free antibody is directly proportional to the mobility difference between the two species: $\Delta L=\left(\Delta \mu / \mu_{A b}\right) L_{A b}$. Both mobility $(\mu)$ and differential mobility $(\Delta \mu / \mu)$ are dependent on PA gel composition according to the Ferguson relationship: $\mu=\mu_{0} 10^{-K \cdot T}$. Here $K$ is the migration retardation coefficient. As the immune complex has a larger retardation coefficient than the antibody due to its larger size, decreasing gel pore-size by increasing $\mathrm{T}$ yields an increased peakto-peak distance $(\Delta L)$ and increased $\mathrm{SR}$ at a given migration distance. However, practical assay implementation requires large pore-size gels in the loading channel to allow unbiased introduction of immune complexes. A $2.5 \% \mathrm{~T}$ large pore-size loading gel was chosen as PA has been observed to fail to crosslink at $\leq 2 \% \mathrm{~T}$. Through photopolymerization, we achieve control over gel pore-size and pore-size distribution in the separation channel for optimal assay performance.

Figure 1A illustrates the uniform large pore-size gels did not resolve immune complex from antibody probe within the first $\mathrm{mm}$ of separation length. In comparison, introduction of a gradual $2.5 \% \mathrm{~T}-$ to- $8 \% \mathrm{~T}$ pore-size gradient in the separation channel (Figure 1B) yields CRP assay completion in $925 \mu \mathrm{m}$ at $12.2 \mathrm{~s}$. Use of the gradient gel yielded separation of anti-TNF antibody from immune 
complex in $850 \mu \mathrm{m}$ at $14.5 \mathrm{~s}$. While the gradient gel notably enhanced the mobility difference between the antibody and complex compared to the uniform large pore-size gels, effective separation of $\mathrm{Ab}^{*}$ from CRP complex was not achieved until the proteins migrated into a pore-size region with $\mathrm{T}>4 \%$. Therefore, increasing the slope of the pore-size gradient, or, equivalently, locating a small pore-size region proximal to the injection junction would result in a more compact device for electrophoretic immunoassays.

Thus, we introduce a nanoporous "step discontinuity" in the separation matrix that yields the shortest separation distances reported to date. Using the nanoporous discontinuity, two-color immunoassays for key inflammation biomarkers (CRP and TNF- $\alpha$ ) require a separation length of just $350 \mu \mathrm{m}$ versus $10 \mathrm{~cm}$ in standard capillary formats typically used for these inflammatory markers. Under full-field imaging mode, quantitation was completed in $4 \mathrm{~s}$ on a discontinuous gel with an equivalent separation gel pore-size of $6 \% \mathrm{~T}$ (Figure 1C). An SR $\geq 1$ was achieved for CRP in a separation length of $330 \mu \mathrm{m}$ while an $\mathrm{SR} \geq 1$ for TNF was achieved in a separation length of $310 \mu \mathrm{m}$. For the discontinuous gel demonstrated here, an applied electric field strength of 100 $\mathrm{V} / \mathrm{cm}$ would require less than $3.5 \mathrm{~V}$ across the required separation length, which is a significant reduction of electrical potential requirement for electrophoretic immunoassays.

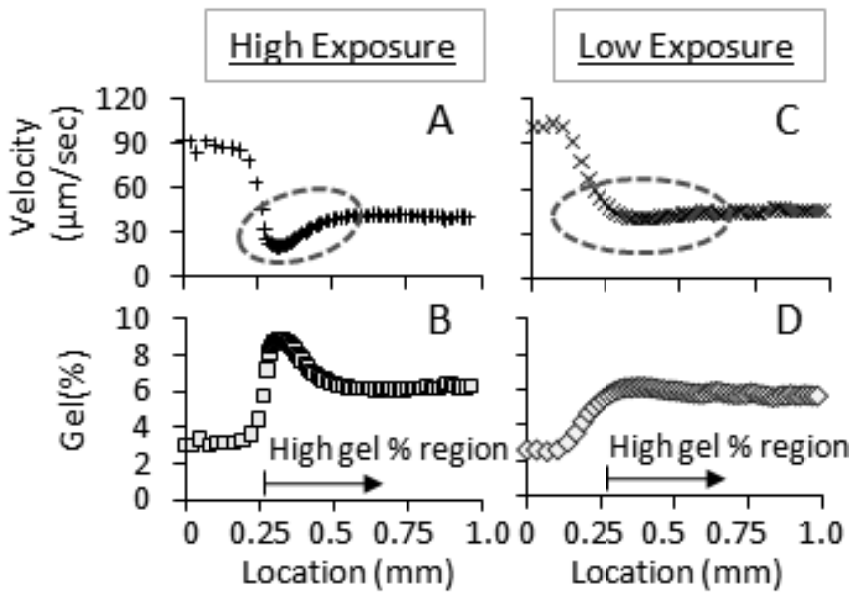

Figure 2. Controlling photopatterning illumination power offers improved control over pore-size at discontinuity. Protein tracer exhibits more uniform velocity aft of discontinuity on $2.5 / 5 \% \mathrm{~T} \mathrm{gel}$ fabricated at $7.5 \mathrm{~mW} / \mathrm{cm}^{2}$ exposure $(C, D) v s . \sim 600 \mathrm{~mW} / \mathrm{cm}^{2}(A, B)$. Both gels were fabricated with $5 \%$ precursor solution in the first exposure. Loading gel $(2.5 \% T)$ fabrication conditions were the same in both cases.

To achieve the desired performance, we have overcome nonidealities that stymied previous attempts by our group and others at discontinuous gel fabrication, namely: (1) exclusion of large proteins [1] and (2) "destacking" of analyte bands during separation [4]. Initial discontinuous gel fabrication using the mercury arc lamp and UV objective as the exposure source yielded a non-ideal pore-size distribution near the discontinuity as revealed by analyte mobility tracking (Figure 2A-B). Exposing a separation channel filled with $5 \% \mathrm{~T}$ precursor solution resulted in an apparent pore-size of $9 \% \mathrm{~T}$ at the discontinuity. The reduced pore-size acted to exclude the larger immune complexes. Additionally, the apparent pore-size increased from that of a $9 \% \mathrm{~T}$ to that of a $6 \% \mathrm{~T}$ gel during the first $300 \mu \mathrm{m}$ aft of the gel discontinuity. The poresize increase resulted in "de-stacking" of analyte bands, negatively impacting separation resolution. We address the performancedegradation by controlling exposure intensity (Fig 2C-D). Illumination power was reduced by inserting metallic-coated neutral density filters in the light path between the mercury arc lamp and the UV objective. With the modified partial-illumination setup and an optimized exposure time of $3.3 \mathrm{~min}$, a more uniform pore-size separation gel can be observed after the interface. An effective pore-size of $6 \% \mathrm{~T}$ near the interface allowed free migration of the immune complexes.

With the optimized fabrication protocol, a $2.5 / 5 \% \mathrm{~T}$ discontinuous gel allows completion of a CRP immunoassay with a nearly $3 \mathrm{x}$ reduction in separation length and assay duration compared to a $2.5 \% \mathrm{~T}$-to- $8 \% \mathrm{~T}$ gradient gel (Figure 3 ). A $2.5 / 8 \% \mathrm{~T}$ discontinuous gel further reduces separation lengths to $<200 \mu \mathrm{m}$ (Fig 3, bottom panel). The $2.5 / 8 \% \mathrm{~T}$ discontinuity excluded the CRP immune complex while allowing antibody migration, thus maximizing the mobility difference $(\Delta \mu / \mu)$. A secondary performance benefit offered by the discontinuous gels is the ability to "stack" proteins, hence improving signal-to-noise ratio and detection sensitivity. The "stacking" effect stems from the slowed migration of the protein analytes across the large-to-small poresize discontinuity. Compared to the $2.5 \% \mathrm{~T}$-to- $8 \% \mathrm{~T}$ gradient gel, the $2.5 / 5 \% \mathrm{~T}$ discontinuous gel achieved nearly 4 times improvement in the signal-to-noise ratio whereas 8 times improvement was observed on the $2.5 / 8 \% \mathrm{~T}$ discontinuous gel (Figure 3, left panels).

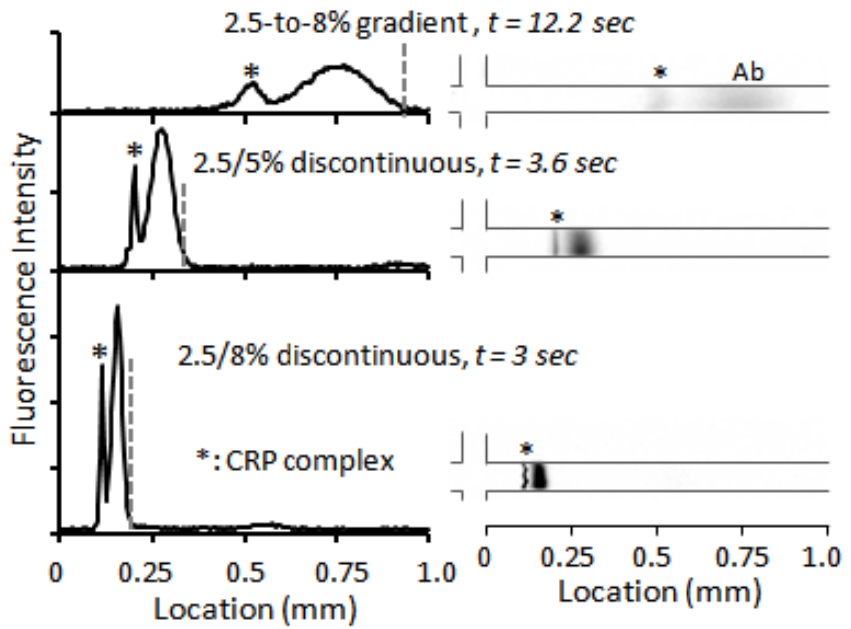

Figure 3. Tuning pore-size of discontinuity achieves baseline resolution in short separation length (indicated by grey dashed line). Channel fluorescence intensity profiles for separation resolution $\geq 1$ are shown on the left for three separation gel formats whereas corresponding CCD separation images are shown on the right. Sample contains 16 nM CRP.

Although the $2.5 / 8 \% \mathrm{~T}$ discontinuous gel resulted in the shortest separation length using full-field imaging for detection, the expense and size of scientific-grade CCD cameras makes PMT or photodetector based single-point detection a preferred method for point-of-care instruments. Under single point detection, assay duration is largely determined by the slowly migrating proteins. If using a $2.5 / 8 \% \mathrm{~T}$ discontinuous gel, the immune complexes could take an infinitely long time to reach the single point detector due to physical exclusion by the small pore-size gels. Therefore, the $2.5 / 5 \% \mathrm{~T}$ discontinuous gel was chosen for single-point detection studies as both TNF- $\alpha$ and CRP immune complexes could enter the separation gel. 
A

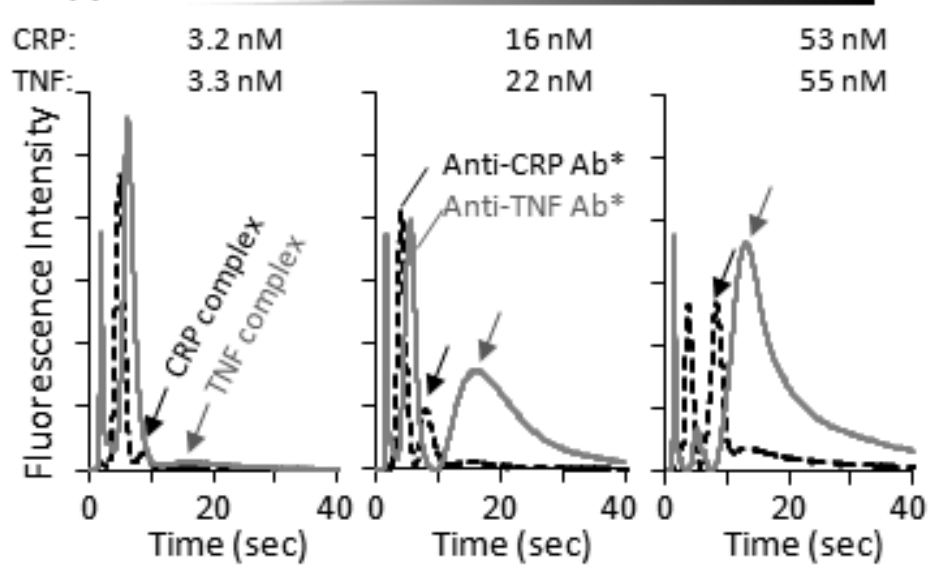

B

CRP: $10.5 \mathrm{nM}$

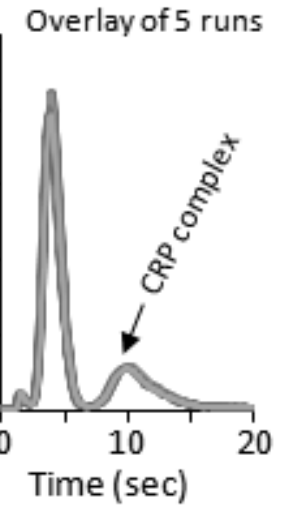

C

CRP: $16 \mathrm{nM}$

in diluted serum

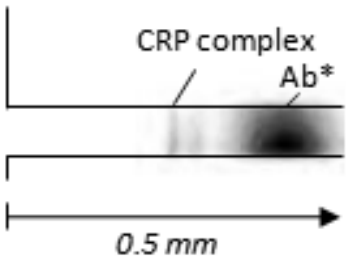

$t=7 \mathrm{sec}$

Figure 4. Inflammation biomarker assay performed on discontinuous gel is quantitative (A) and reproducible (B). (A) Electrophoregrams generated on discontinuous gel chip with incubates containing $C R P=3.2 n M / T N F=3.3 n M, C R P=16 n M / T N F=$ $22 n M, C R P=53 n M / T N F=55 n M$. (B) Electrophoregrams from the $1^{\text {st }}, 3^{\text {rd }}, 5^{\text {th }}, 7^{\text {th }}$, $9^{\text {th }}$ run over a 20 min period are overlaid. (C) illustrates CRP measurement performed in diluted serum.

Simultaneous quantitation of TNF- $\alpha$ and CRP were performed on the $2.5 / 5 \% \mathrm{~T}$ discontinuous gel. Red and green fluorescence collected just $270 \mu \mathrm{m}$ downstream of the injection junction was spectrally demultiplexed via bandpass filters followed by PMT detection (Figure 4A). Both CRP and TNF- $\alpha$ assays are quantitative, with increasing biomarker concentration resulting in increased complex peak areas and a commensurate decrease in free antibody. In contrast to surface-immobilized biosensors, continuous monitoring is feasible via sequential injections and separations. For ten sequential CRP assays conducted over a 20 min period (Figure 4B), the analyte migration times exhibited a \%RSD less than $2.5 \%$. The \%RSD for the SR was $1.0 \%$ and the antibody-to-complex peak height ratio exhibited a $2.7 \%$ RSD. The reproducibility performance is expected because polyacrylamide gels suppress non-specific adsorption and the separation gel poresize was optimized for unrestricted migration of analytes of interests. Detection of CRP in diluted human serum was demonstrated in $7 \mathrm{~s}$ within $500 \mu \mathrm{m}$ of separation length; analysis of additional samples is underway (Fig 4C).

\section{CONCLUSIONS}

With the introduction of discontinuous nanoporous gels, electrophoretic immunoassays are now amenable to low voltage $(<$ $9 \mathrm{~V})$ operation while maintaining high electric fields $(>100 \mathrm{~V} / \mathrm{cm})$ due to the ultra-short separation lengths $(<0.5 \mathrm{~mm})$. Demonstrated here for two inflammatory markers (including CRP in human serum), the assay formats are adaptable for other protein biomarkers through tuning sieving matrix pore-size to match target analyte mobilities. Our current efforts focus on revisions to chip designs to realize portable diagnostic instruments and assessment of clinical samples for translation to near-patient environments.

\section{ACKNOWLEDGEMENTS}

The authors thank the Hellman Family Faculty Award at UC Berkeley for generous financial support, as well as Caliper Life Sciences for glass chip and equipment support. The authors thank Ms. Kelly Karns and Dr. Mei He for assistance. C. Hou is a National Science Foundation Graduate Research Fellow. A. E. Herr is a DARPA young faculty awardee and Alfred P. Sloan Research fellow in chemistry.

\section{REFERENCES}

[1] A. E. Herr, D. J. Throckmorton, A. A. Davenport, and A. K. Singh, "On-chip native gel electrophoresis-based immunoassays for tetanus antibody and toxin," Analytical Chemistry, vol. 77, pp. 585-590, Jan 2005. C. Hou and A. E. Herr, "Multiplexed analysis of inflammation biomarkers using spectrally-encoded onchip electrophoresis," in Transducer 2009, Denver, CO, 2009, pp. 1574-1577.

[3] C. T. Lo, D. J. Throckmorton, A. K. Singh, and A. E. Herr, "Photopolymerized diffusion-defined polyacrylamide gradient gels for on-chip protein sizing," Lab on a Chip, vol. 8, pp. 1273-1279, Aug 2008.

[4] S. Yang, J. K. Liu, C. S. Lee, and D. L. Devoe, "Microfluidic 2-D PAGE using multifunctional in situ polyacrylamide gels and discontinuous buffers," Lab on a Chip, vol. 9, pp. 592-599, 2009.

\section{CONTACT}

*Amy E. Herr, tel: +1-510-6663396; aeh@berkeley.edu 